Review

\title{
Slim Accretion Disks: Theory and Observational Consequences
}

\author{
Bozena Czerny \\ Center for Theoretical Physics, Polish Academy of Sciences, Al. Lotnikow 32/46, 02-668 Warsaw, Poland; \\ bcz@cft.edu.pl
}

Received: 15 April 2019; Accepted: 21 May 2019; Published: 26 May 2019

\begin{abstract}
The concept of slim accretion disks emerged over 30 years ago as an answer to several unsolved problems. Since that time there has been a tremendous increase in the amount of observational data where this model applies. However, many critical issues on the theoretical side remain unsolved, as they are inherently difficult. This is the issue of the disk stability under radiation pressure, the role of the magnetic field in the energy transfer inside the disk, the formation (or not) of a warm corona, and outflows. Thus the progress has to be done both through further developments of the model and through careful comparison with the observational data.
\end{abstract}

Keywords: black hole; accretion disks; slim disks; accretion disk stability

\section{Introduction}

Slim accretion disk models were proposed in 1988 by Abramowicz et al. [1]. They describe an optically thick, geometrically not very thin quasi-Keplerian accretion flow onto a black hole in high-Eddington-ratio sources. In such a flow, a considerable part of the energy dissipated in the disk interior is carried radially with the flow instead of being reemitted at the same radius, as in the standard Shakura and Sunyaev [2] accretion disk model. Observed sources are mostly sub-Eddington, but a fraction of active galactic nuclei and some galactic binaries during some stages are close to or above the Eddington ratio, and the slim disk model should apply there. Observations indeed support the presence of the optically thick disks in these sources. However, important issues related to the stability of these models and necessary modifications is still under debate. The slim disk of Abramowicz et al. [1] is based on the so-called $\alpha$-viscosity assumption, while a fully self-consistent model should predict the viscous torque. Three-dimensional numerical magneto-hydrodynamical simulations do not yet have all the necessary ingredients to realistically show how the flow onto the black hole proceeds, although there has been tremendous progress in this direction. Thus, at this moment, semi-analytical models are also very useful in confronting the models with observational data. One of the key issues is actually the presence of heartbeat states in some astronomical sources and their relation (or not) with the limit-cycle behavior predicted by the slim disk model.

\section{Accretion Disk Models}

The key parameters in the process of accretion onto a black hole are the angular momentum of the inflowing material and the accretion rate. Here we concentrate on high angular momentum flow, which leads to the formation of an accretion disk, and the azimuthal velocity of this flow at a given radius is of Keplerian order, apart from the innermost region of the flow close to the innermost stable circular orbit (ISCO). 
The accretion rate is conveniently expressed using the dimensionless quantity, $\dot{m}$, the ratio of the accretion rate to the Eddington accretion rate, $\dot{M}_{E d d}$. The formal definition of the latter quantity is

$$
\dot{M}_{E d d}=\frac{L_{E d d}}{\eta c^{2}}
$$

where the Eddington luminosity is defined as usual (for a spherically symmetric setup, pure hydrogen plasma):

$$
L_{E d d}=\frac{4 \pi G M m_{p} c}{\sigma_{T}},
$$

where $M$ is the black hole mass, $c$ is the speed of light, $\sigma_{T}$ is Thomson cross section for scattering in a fully ionized material, $m_{p}$ is the proton mass, and $\eta$ is the dimensionless accretion efficiency.

Here the subtle point is that the accretion flow efficiency of a slim disk itself depends on the accretion rate. In the model of Shakura and Sunyaev [2], the accretion efficiency is $1 / 12$. In the full GR extension of this model [3], it is uniquely given by the spin of the black hole, but in slim disks part of the energy is trapped, and as the accretion rate rises, the fraction advected under the horizon rises, and the efficiency of accretion flow drops. Therefore, some authors use $\eta=1$. in Equation (1), some use the "typical value" of 0.1 , and finally some indeed calculate it from the model. In the discussion below I will generally use $\eta=0.1$ as a reference value.

The currently used accretion disk models are thus divided predominantly according to the Eddington ratio of the flow, although the value of the angular momentum at the outer disk (more precisely, its relation to the local Keplerian angular momentum) also plays a role. The physics of the accretion process is well-described in a number of reviews (e.g., [4]), and the best introduction in book form is offered by Frank et al. [5]. Here I shortly summarize the basic forms of accretion flows.

\subsection{Spherical Accretion and Low-Angular Accretion Flow}

Spherical accretion flow has negligible angular momentum, and the best example is Bondi flow. A significant part of the flow proceeds supersonically. The flow can be optically thin, as in the case of accretion in weakly active galaxies, or it can be very optically thick as in the case of the dynamical collapse of a star. If some angular momentum is present, the material meets the centrifugal barrier. Accretion can proceed with or without a shock formation. If the corresponding circularization radius is much larger than the innermost stable circular orbit (ISCO), the rest of the flow has to be described by another model.

\subsection{Optically Thin Advection-Dominated Accretion Flow (ADAF)}

If the Eddington ratio is low (well below a few percent) and the angular momentum is a fraction of the Keplerian angular momentum, the flow is optically thin, the ion temperature is close to the virial temperature, and the electron temperature (much smaller than the ion temperature in the innermost part of the flow) is determined by the interaction with ions and direct dissipation mechanisms heating electrons. Such a flow is characteristic for low-luminosity active galactic nuclei (AGN), and for very low-luminosity states in galactic sources. A significant fraction of the energy is then advected towards the black hole (hence advection-dominated accretion flow, ADAF). Since the electron temperature there is high, the flow is an efficient source of $\mathrm{X}$-ray emission.

\subsection{Standard Optically Thick Geometrical Disk}

When the Eddington ratio is higher, the disk material cools down efficiently due to higher density, and the flow becomes optically thick, emitting locally as a black body to a good approximation. The angular momentum of such flow is locally Keplerian Shakura and Sunyaev [2] (for the full general relativity version see Novikov and Thorne [3]). The disk emission dominates the UV band (for AGN) and soft X-ray band (for galactic sources). The inner radius of the flow is well described by the position 
of the ISCO. Heat advection is negligible in these models, and the local emissivity is by the local dissipation enforced by the transfer of the angular momentum. The accretion efficiency of the flow is set by the position of ISCO (i.e., by the black hole spin). Such models apply well to (some) soft states in galactic sources, and to many quasars in the optical/UV band.

\subsection{Slim Disks}

When the accretion rate becomes higher than the Eddington rate, the assumptions underlying the standard model break down. The advection term becomes important, the inner radius of the disk moves from its ISCO position (mostly inward, but the effect depends on the viscosity), and the departures from the Keplerian angular momentum due to the presence of a strong radial pressure gradient become important. The local emission is no longer given by the local dissipation, and a fraction of energy is lost through the inflow under the black hole horizon. The accretion efficiency is lower than in the standard disk, and it decreases with the rise of the accretion rate. These models apply to super-Eddington quasars, Narrow Line Seyfert 1 galaxies, to some phases of gamma-ray bursts (but then the physics has to be modified to include neutrino cooling and nuclear processes), and to tidal disruption events (TDEs).

\subsection{Transitions between the Models}

Here slim disks and standard disks belong to a single branch of solutions. Full slim disk equations can be used to describe the standard disks. The additional terms present in the slim disk automatically become relatively unimportant when the accretion rate is well below the Eddington rate. This is not the case for the transitions between optically thin and optically thick models. If the viscosity parameter is very high (close to or above 1), a formal bridge between ADAF and slim disk is found (skipping the standard gas-dominated disk stage), but for lower (more realistic) values the two branches are separate, as shown already in Abramowicz et al. [6]. Thus, the transition between the standard cold disk and the inner ADAF is still under debate, and it seems to require more physics (e.g., electron conduction) to describe this process $[7,8]$, although attempts at describing this transition just on the basis of heating/cooling change have also been made [9].

\section{Historical Remarks about Slim Disk Model Formulation}

The slim disk concept developed out of three earlier independent lines of study which later converged into a self-consistent and practical model.

The oldest line of research focused on the proper description of the inner boundary condition of a standard Keplerian disk. The classical model of Shakura and Sunyaev [2] assumed that there is no torque acting at the inner edge of the flow and concentrated on the description of the disk at larger radii, not even touching on the issue of how the flow actually proceeds from the disk toward the black hole horizon. The issue was raised in a number of papers, but without offering a satisfactory solution [10]. Led by Bohdan Paczynski, we started the research in this direction at the Copernicus Astronomical Center, starting from the proper understanding that the flow must actually be the same as the outflow through the inner Lagrange point L1 in binary starts. Paczynski and Bisnovatyi-Kogan [11] used a simplified version of equations which did not yet allow description of the transition of the flow from subsonic outside to supersonic flow below the innermost stable circular orbit. Loska [12] defined conditions of the transonic flow in the case of barotropic gas, and the complete description of the flow, with advection term, radial radiative term, and equation of state based on a self-consistently determined ratio of gas to radiation pressure was presented by Muchotrzeb and Paczynski [13]. The model described using the vertically averaged disk model and the gravity field of the black hole was described in pseudo-Newtonian approximation [14]. The solution had to be determined numerically, and the code prepared by Muchotrzeb and Paczynski [13] was used by Abramowicz et al. [1] as it already had all the required elements, and it already implied that with the rise of the accretion 
rate for a given black hole mass, the sonic point moves inward from the ISCO (innermost stable circular orbit) if the viscosity coefficient is small (for large-viscosity behavior, see Muchotrzeb [15]).

The second line of research concentrated on geometrically thick accretion disks, known as Polish doughnuts, which were seemingly required to produce well-collimated extended jets observed in a fraction of active galaxies. This line of research was also vigorously studied in Warsaw [16]. These models were truly two-dimensional, optically thick disks, and their construction was strongly based on the general idea of figures of equilibrium. For example, models created by Paczyński and Wiita [14] using the pseudo-Newtonian potential had an inner as well as outer edge. Their radial extension was determined by the adopted radial distribution of the angular momentum, since the inner and the outer edges were located at the interception points with the Keplerian angular momentum distribution. Finding the equipotential surface passing through the inner and outer radius allowed the full 2-D shape to be obtained immediately. The local radiation from the disk surface was calculated assuming that the local radiation flux perpendicular to the disk surface is equal to the Eddington flux. Thus, overall emission corresponded to super-Eddington luminosity due to geometry. The global energy budget provided the corresponding accretion rate. However, the local energy budget between heating and cooling is not considered in these models, and therefore the angular momentum distribution has to be assumed. In slim disks (developed later), this budget is considered, and therefore the angular momentum distribution is calculated self-consistently for an assumed accretion rate. These models are not used to interpret the data since they require these additional assumptions, but they are useful as an initial setup for MHD time-dependent computations. An important contribution here to the slim disk concept was also the observation that at a certain regime (above the Eddington limit) the radiation in the (spherically symmetric) flow is trapped $[17,18]$. It also showed a geometrical similarity between the inner edge of a geometrically thick disk and a star filling its Roche lobe.

The third line of research was devoted to the problem of the thermal and viscous instability of a standard disk. These two instabilities were discovered (soon after the publication of Shakura and Sunyaev [2]) by Pringle et al. [19] and Lightman and Eardley [20], correspondingly (for the combined effect of the two instabilities, see Shakura and Sunyaev [21]). This questioned the very existence of the accretion disks, otherwise attractive as an explanation of the accretion phenomenon in binary systems and in active galactic nuclei [2,22]. The use of unstable models seemed problematic. The way out was first searched for in the form of modification of the viscosity law. The classical model of Shakura and Sunyaev [2] is based on the assumption that the viscous torque is proportional to the total pressure in the disk interior (i.e., gas plus radiation pressure), and the instability appeared for relatively high accretion rates, when the radiation pressure starts to dominate. Sakimoto and Coroniti [23] proposed a pure magnetic viscosity model and argued that in this case the viscous torque is proportional only to the gas pressure, which led to stable disks of much higher surface density, with self-gravitational instability in the outer parts. Abramowicz et al. [1], introducing the slim disk model still adopted the viscosity prescription of Shakura and Sunyaev [2], but included the advection term which acts (predominantly) as a cooling term. Thus, the disk becomes stable at very high accretion rates. This discovery brought back the interest in accretion disk models as the models of the continuum emission in binary black holes and active galactic nuclei.

\section{Applicability of Slim Disks and Their Observational Appearance}

The slim disk equations in their original formulation $[1,13]$ were using the pseudo-Newtonian gravitational potential of Paczyński and Wiita [14], and they used the vertically averaged disk structure. However, they contained the following key elements: the advection term, the radial pressure gradients, and the proper description of the inner boundary conditions. The presence of the radial pressure gradient allowed the determination, instead of just the assumption, of the radial angular momentum distribution of the angular momentum in the disk. Specifically, the presence of these terms allows determination of where the radial velocity of the flow becomes supersonic, and it must become supersonic before it crosses the black hole horizon. As an inner boundary, the zero-torque condition 
is assumed, not at the ISCO as in the standard disk, but at the black hole horizon. The flow is thus calculated all the way to the black hole horizon, and the location of the sonic point (usually below the ISCO) results from the model and depends on the accretion rate and viscosity. Due to the presence of these additional radial terms, the model also describes the heat advected with the flow. As mentioned before, for accretion rates much lower than the Eddington ratio the model is in practice similar to the standard model, the advection term is almost unimportant (apart from the region where the flow changes into supersonic), but this transition happens very close to the ISCO, and not much energy is dissipated there.

Further numerical developments cover the formulation of the slim model in full GR, which gives the opportunity to study the dependence of the model on the black hole spin [24] since the pseudo-Newtonian approach only mimics the Schwarzschild solution well. In addition, Sagdowski et al. [24] also introduced a description of the vertical structure of the disk by combining slim disk equations in the vertical plane with the disk vertical structure equations from Newtonian model of RóżaŃska et al. [25] which include the vertical radiative transfer, convection in the vertical direction, and local description of the heating using the $\alpha$ parameters. This $1+1$ structure is the most advanced stationary slim model built to date. However, many trends can be studied using much simpler models, such as semi-analytical models like super-critical self-similar solutions [26].

The role of advection in the disk rises with the rise of the accretion rate, $\dot{m}$, and some effects start to be seen at $\dot{m}=0.3$, and the effects are very important for $\dot{m}$ above 1 . The advection modifies the shape of the accretion disk spectrum. The long wavelength range is not strongly affected since this part of the emission comes from the outer parts where advection is not yet strong, but the innermost part of the disk emits less than in classical model, so the slim disk spectrum is redder than the corresponding standard model. Finally, the radiation flux emitted close to the ISCO in slim disk is again enhanced (see Figure 7 in [13]), but this affects the total spectrum less, since only a relatively small fraction of energy is dissipated there. The effects were studied in a number of papers, and models were applied to a whole range of sources from binary stars, through intermediate black hole mass candidates to active galaxies Szuszkiewicz et al. [27], Straub et al. [28]. However, observationally the issue is not quite settled, for example recent work on fitting broad band spectra of high-Eddington-ratio and lower-Eddington-ratio sources did not show any clear differences, and both were well-fitted by a standard disk model [29]. The improvement in use of the slim disk over the standard disk was also not very significant in the case of binary systems close to the Eddington luminosity [30], although the model used there—developed by Sądowski et al. [24]—was already an improvement of the original model of Abramowicz et al. [1].

The slim disk model predicts that as the accretion rate rises, the luminosity rises relatively slowly, and finally saturates at the value of about 10 times higher than the Eddington luminosity. The luminosity rise with accretion rate (see e.g., Figure 11 in Abramowicz and Fragile [4]) above the Eddington rate can be well approximated as a logarithmic rise

$$
L=2 L_{E d d}(1+\ln (\dot{m} / 5))
$$

for a non-rotating black hole [31].

AGN approaching this limit were thus proposed to be used as standard candles in cosmology to measure the expansion rate of the Universe [32,33].

Accretion disks at high accretion rate become geometrically thicker, which additionally collimate the radiation emitted by the innermost part of the disk and affect the illumination of the disk's surroundings, including the broad-line region [31].

Determination of the black hole mass and Eddington ratio in large quasar samples are never very accurate; most of the higher-quality measurements from the catalog of Shen et al. [34] imply that most quasars concentrate around $L / L_{E d d} \sim 0.1$, but the tail goes to or above the Eddington ratio [35]. Eddington or super-Eddington accretion rates were claimed for a number of sources (e.g., [36-39]), and the results or the reverberation measurements in super-Eddington AGN were different from 
similar results for sources with lower Eddington ratio [40,41], supporting the view that a fraction of the sources indeed belong to this category.

\section{Stability of Classical Slim Accretion Disks}

Although the aim of formulating the slim disk model was to provide a stable solution for the high-accretion-rate flow, this goal was not actually fully achieved. However, the most important step was made in answering the question: what happens when the disk becomes unstable due to radiation pressure? The answer was: at sufficiently high accretion rate, the flow cools down by advection and the flow is stabilized. However, the global picture is more complex, in close analogy to the ionization instability issue in the case of cataclysmic variables and X-ray novae.

The global behavior of the accretion disk is governed by two trends: one describing the local behavior of the disk structure changes with the rise of the accretion rate, and the second which shows how this behavior scales with the distance from the black hole.

It we study the disk structure close to a black hole locally, for example at $10 R_{S c h w}$ (where $R_{S c h w}=$ $2 G M / c^{2}$ ) as a function of the accretion rate, at low accretion rate the disk surface density rises, when the pressure is dominated by the gas pressure. With further rise of the accretion rate we enter the radiation-pressure-dominated branch, and the disk surface density decreases with the rise of the accretion rate. This solution is unstable, since the heating rises more rapidly than the disk cooling, with a small rise in the disk temperature. Finally, the advection sets in when we approach the Eddington limit, and a new stable advection-dominated branch develops. Now again the surface density in the disk rises with the increase of the accretion rate. Thus, the disk becomes stable for high accretion rates. This is quantitatively illustrated in Figure 2 of Abramowicz et al. [1], and is schematically shown in Figure 1 above, first insert. Since the $\dot{M}-\Sigma$ plots show the stability or instability of a particular disk so nicely, we call these plots stability curves.

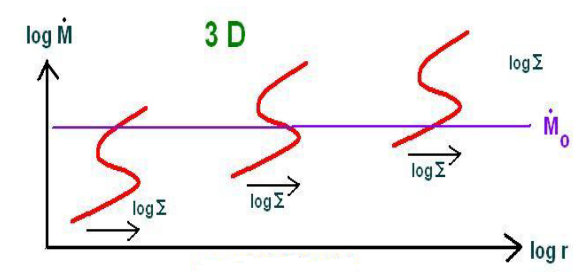

Figure 1. Schematic illustration of the stability curve of an optically thick disk at a few distances from a black hole. A single $\Sigma-\dot{M}$ picture shows the dependence of the disk surface density, $\Sigma$, on the accretion rate $\dot{M}$. The positive slope describes the stable branch (gas-pressure-dominated lower branch, advection-dominated upper branch), the intermediate radiation-pressure-dominated branch is unstable. The whole curve position shifts up with increasing radius, so when we look the location where the external assumed accretion rate crosses the plots we see (for high accretion rate) an inner, stable advection-dominated branch, an intermediate unstable zone, and an outer stable gas-dominated disk.

Abramowicz et al. [1] plotted the stability curves only in a very narrow range of radii. The upper branch of this curve (stable branch) corresponds to a slim disk solution while the other two branches are predicted by the classical disk model. The intermediate branch is unstable. In [2] this branch would extend until an arbitrary accretion rate, but the new terms present in the slim disk model (negligible at low accretion rates) force the unstable branch to bent. However, if we go to radii on the order of a few hundred $R_{S c h w}$, the overall shape of the curve does not change much, but the "location" of the curve changes dramatically. This is schematically illustrated in Figure 1. The dominating effect is the shift up, so if we consider an accretion rate of a few (in dimensionless units, introduced above), at a distance of hundreds of $R_{S c h w}$ this accretion rate would imply that the disk is at the unstable radiation-pressure-dominated branch, and advection is still unimportant. Meanwhile, if we go further from the black hole, for the same accretion rate the disk is still dominated by the gas pressure. This happens since in the classical solution of Shakura and Sunyaev [2], the ratio of the radiation to 
gas pressure generally goes down with the radius for a fixed accretion rate. The radii when these transitions take place depend on the mass of the central body, and in general the accretion disks in active galaxies are much more radiation-pressure-dominated than in the case of accretion disks at binary black holes. The stabilization due to advection happens in both at roughly the same accretion rate, but the stable and unstable zones are much more extended in active galaxies, as illustrated in Janiuk and Czerny [42].

Therefore, unless the outer radius of the disk is very small, slim disks always have an intermediate unstable zone. Thus, the models are not stationary. Still, due to the new advection term it was later possible to calculate the time evolution of the disk in the same way as was done for cataclysmic variables $[43,44]$, where the stable upper and lower branches were provided by neutral and fully ionized disk states, complementing the intermediate unstable branch corresponding to partially ionized gas. Only for a slim disk do we have an upper advection-dominated branch and lower gas-pressure-dominated branch. If at a given radius the mean accretion rate locates the disk on the unstable (radiation-pressure-dominated) branch, the disk structure performs the time evolution, with episodes of outbursts and low states. During outburst, the source is on the upper branch, the temperature and radiation flux are high, and the accretion rate is high, but the ring effectively loses material, as the mass inflow towards the black hole is not fully compensated by the inflow from outer radii. The disk surface density systematically decreases during this phase. At low state, the temperature and the radiation flux are low, the material effectively accumulates in the ring, and the surface density rises slowly in a viscous timescale. The limit cycle must be happening anti-clockwise on the stability curve, as the arguments above imply. The transition from the upper to lower branch takes place when the disk cannot achieve a solution in thermal balance on the upper branch with further reduction of the surface density, and the transition takes place in the thermal timescale. Roughly the same happens when the ring accumulates so much mass that further increase of the surface density leads to lost thermal balance and the disk rapidly expands, reaching the upper branch.

Of course, rings are coupled, so it is actually necessary to calculate the evolution of the whole disk. However, it looks qualitatively similar: large parts of the disk alternate between the upper and the lower stable branches. The inner, formally stable, part of a slim disk is also affected since the outer-unstable-part of the disk alternates the supply of the material to the innermost part. So, the whole inner disk is subject to strong variability.

The models of the time-dependent evolution have been calculated by a number of authors [45-51], generally with the aim of comparing with some observational data for objects showing outbursts. Thus, in order to fit the outburst amplitude, some modifications of the model are usually introduced since generally the outbursts calculated without any modification had amplitudes that were too high. We will return to this issue later on. In general, these simulations showed regular or semi-regular changes of the predicted luminosity up to a few orders of magnitude. Computations were done in 1-D approximation, but globally, with calculated total duration of several of the viscous timescales at the stable outer part of the accretion disk. However, they still resolved the time evolution of the inner disk part in the thermal timescale.

An important point was raised by $\mathrm{Gu}$ and $\mathrm{Lu}$ [52]. The equations of the slim disks were always solved in a geometrically thin disk approximation (i.e., with expansion of the gravitational potential against $z / r$, where $z$ is the distance from the equatorial plane). This might not be correct for sources with high Eddington ratio, since the prediction of the standard disk is that the disk thickness in the radiation-pressure-dominated part increases linearly with the accretion rate [2]. If the local disk thickness become larger than the radius, the gravity force in the vertical direction would start to decrease with further increment of the accretion rate. This in turn might lead to a strong outflow. However, Lasota et al. [53] argued that this never happens since the advection term will prevent the slim disk from becoming thick at an arbitrary high accretion rate. Thus, the standard computations of the slim disk evolution are self-consistent. 


\section{Observational Support of Outbursts Due to Radiation Pressure}

The issue is still under debate as to whether some regular outbursts observed in accreting sources are actually signatures of the radiation pressure instability in slim disks. In the class of binary black holes, two sources display well-defined periodic outbursts (now referred to as heartbeat mode): GRS 1915+105 and IGR J17091-3624. The outbursts last on the order of seconds to hundreds of seconds. Several properties of the outbursts are well-modeled by the radiation pressure instability. Transitions between the states take place anti-clockwise [47], and outbursts are longer when the mean accretion rate is higher [46]. Additionally, the time delay between the hard X-rays and the soft X-rays is in agreement with the data if a model of an unstable disk with an accreting corona is used for GRS 1915+105 [54]. On the other hand, other sources do not show such clear states, even at high Eddington ratios, which can either be interpreted as an argument against the very existence of the radiation pressure instability, or might imply that in other sources the effect of winds and/or magnetic field changes the regular outbursts into semi-regular periods of enhanced variability Janiuk and Czerny [42].

Active galaxies are much larger in size, so the timescales of the same phenomenon are correspondingly longer, and the predicted durations of outburst caused by radiation pressure are on the order of thousands of years, and they are not accessible to direct observations. However, statistical comparison of the duration of the active phase in short-lived radio-loud AGN is generally consistent with expectations of the slim disk instability [49,55]. Wu et al. [56] argued that this is a universal phenomenon seen across the whole black hole mass scale, from binary systems through intermediate black hole outbursts (source HLX-1) to active galaxies.

The comparison of the simple parametric models with the data is one of the ways to establish whether this instability is present. On the other hand, models are based on the assumption of the viscosity law, and some modifications are usually required to decrease the outburst amplitude, like the use of the $\alpha \sqrt{P_{\text {gas }} P_{\text {tot }}}$ or even more general models (e.g., [50,57]), or wind coupled to the local accretion rate (e.g., [58]).

\section{MHD Simulations of Radiation-Pressure-Dominated Disks}

Ohsuga et al. [59] were the first to calculate the model of the supercritical accretion flow. The flow settled down to a quasi-stationary state. However, these computations were still done in radiative hydrodynamical (RHD) mode, and so used the viscosity prescription basically as in a standard disk. In addition, the radial range considered was too small to see any issues related to the global stability discussed above (input material had a circularization radius at $100 r_{g}$ ).

More importantly, since the year 1981 we have been convinced that the viscosity is generated as a result of magnetorotational instability (MRI; Balbus and Hawley [60]). Therefore, the ad-hoc assumption of the $\alpha$-viscosity law is no longer needed (in principle). The real progress and answer to the question about the global stability of the super-Eddington flow should come from radiative MHD computations. With this insight, the arbitrary assumption of the viscous torque scaling is not needed, as it should come automatically and self-consistently from the computations.

However, in reality the issue is not as simple as outlined above. If MRI instability has to be followed, the computations of the disk structure must be done in 3-D (even 2-D is not a good approximation for proper description of the dynamo action). The computational timestep must also be shorter than in the 1-D computations discussed in Section 5. So, instead of a single PC, computations are to be run in computer clusters, and they have difficulties in covering the whole disk for many viscous timescales.

Most 3-D MHD simulations are actually performed in a shearing box approximation, when only a very narrow radial zone is included and periodic boundaries are assumed. This apparently affects the results. First results suggested that radiation pressure instability does not develop in real radiation-pressure-dominated disks [61,62], but subsequent simulations using larger grid domain and more advanced radiative transfer approximation were able to see the thermal runaway after a few thermal timescales [63]. 
Jiang et al. [64] concluded that the disk is stabilized if the opacity in the computations include the atomic transitions of iron ions instead of just Thomson cross section, but again they used a very narrow ring in their computation and the global stabilization of the disk by the use of more complex opacity is unlikely [51].

\section{Hot Coronae, Warm Coronae, Outflows}

The most important issue of the slim disk model and its limitations are related to topics we still do not understand, and observations show us clearly that our knowledge is incomplete.

Like the classical disks, slim disks do not provide an explanation for the jet formation, hard X-ray emission, soft X-ray excess, or winds. This is because the disk is basically assumed to be dominated by the thermal pressure, and MRI can change it into outer optically thin layers which do not contribute much to the total spectrum. On the other hand, spectra and variability imply that that these phenomena take place in the inner part of the disk. So, something must be missed in the model.

Adding these elements to the model parametrically modifies the behavior of the disk itself, including the disk stability. Winds can reduce the outburst magnitude, as already mentioned in Section 5, but the magnetic field providing the energy transport deep inside the disk can completely stabilize the disk. Such models in parametric form were proposed by a number of authors, particularly in the context of the corona formation and hot flow/cold flow transitions [25,65-69]. More advanced but still semi-analytical models of these phenomena were recently developed, for example by Begelman et al. [70], Begelman and Silk [71], Dexter and Begelman [72], and Gronkiewicz and Różańska [73]. Some 3-D simulations of such flows were also performed [74], but they required the ad-hoc presence of the large-scale magnetic fields which did not result from the simulations.

More complex time-dependent behavior of the disk interior can also stabilize the disk. Janiuk and Misra [75] considered the role of the stochastic fluctuations of the disk which are naturally expected due to the MRI-based viscosity mechanism. They showed that a sufficiently large amplitude of such turbulence quenches the radiation pressure instability. This model is semi-analytical, and in principle, the described behavior should be reproduced in advanced MHD computations without any ad hoc assumptions. However, current MHD results are not yet capable of reproducing many other phenomena (e.g., power spectra, frequency-dependent time lags), which still have to be modeled alternatively [76].

One interesting possibility is related to the idea of magnetically arrested disks (MADs), which could lead to the modification of flow close to the central object due to the accumulated strength of the large-scale field lines. This phenomenon has been considered by a number of authors $[77,78]$, and it could be responsible for the radio loud/radio quiet dichotomy in active galaxies, for the phenomenon of changing look (CL) AGN, or for the appearance of ultra-luminous X-ray sources. However, MAD simulations have generally been done in the context of optically thin ADAF-type flow, and not in the case of slim disks, and we do not know if the same mechanism can also work for those disks.

\section{Missing Physics}

We may still be missing some key elements in the global 3-D MHD simulations. We do not know whether it is realistic to expect that a basic MHD run of the accretion process will explain all the complexity observed in accreting black holes. However, this is certainly a goal, and we should at least try to test if we have enough physics in the equations to expect that. Some elements are still clearly missing.

For example, most recent 3-D simulations performed so far for sub-Eddington accretion rates by Jiang et al. [79] include new elements, such as radiation viscosity in the optically thin corona region. This allows them to see the formation of warm/hot corona, with temperature above $10^{8} \mathrm{~K}$ in the innermost part of the disk, and the corona becomes more compact for rising accretion rate. 
Ghoreyshi and Shadmehri [80] propose the addition of the radial viscous force. McKinney et al. [81] show that double Compton and cyclo-synchrotron cooling is also important and affects the conclusions about the temperature in the innermost part of a slim disk. Convection is certainly present in the accretion disks [25,82]. This should automatically be present in global 3-D simulations, but not in shearing-box approximation.

Several processes are well-known, but usually not included in the modeling due to their complexity. These include electron conduction [83] and magnetic field reconnection [84].

We may also need to return to the issue of the anomalous viscosity in the disks (i.e., $\alpha$-viscosity). It is nicely summarized by Hawley and Balbus [85]. They stress that the standard dynamical viscosity in the flow, $v$, when forced to provide the requested $\alpha$-viscosity has implications for the mean free path and the turbulent velocity of the medium:

$$
v=\rho v_{t} L=\alpha \rho v_{s} H,
$$

where $\rho$ is the local density, $v_{t}$ is the turbulent motion velocity, $v_{s}$ is the speed of sound, $L$ is the mean free path of a particle, and $h$ is the disk thickness. $\alpha$ is on the order of 0.1 in the model of Shakura and Sunyaev [2]. This means that we usually assume a mean free path of the particle on the order of $0.1 \mathrm{H}$, and the turbulent motion velocity equal to that of the speed of sound. This is extreme, and is why the viscosity operating in accretion disks was named "anomalous". MRI solved this problem; we no longer need "anomalous" viscosity. However, we should note that if a hot two-temperature corona forms above an accretion disk, in such a corona the mean free path of ions is actually quite large, and the speed of sound is large there as well. Therefore, standard dynamical viscosity should effectively provide an efficient accretion flow in the hot corona. This is not yet included in any 3-D simulations to my knowledge.

\section{Conclusions}

In this review I shortly presented the history of the slim disk model, its applicability range, and most of all, its limitations. The description of the accretion flow close to or above the Eddington limit is still quite uncertain. Further, more advanced MHD simulations are needed, but they have to be supplemented with simpler, easy to calculate 1-D models that can be easily compared to the broad-band observational data for galactic binary systems and active galaxies. Modeling time dependence in observed sources is particularly valuable since stationary spectra are frequently rather degenerate with respect to the model properties. We should keep in mind that the spectral properties of a stationary, optically thick, geometrically thin Keplerian disk of [2] do not depend on the disk interior (including the viscosity law) while the time-dependent evolution reflects the internal structure. Slim disks should also be tested not only against the spectral properties but also through their time variability. A parametric approach to modeling the data can also help us to know how frequently real objects are in this regime, so the development of models with the slim disk option included [86] and which are prepared to be fitted to the data is very valuable.

Funding: This research was partially supported by the National Science Center, Poland, grant No. 2017/26/A/ ST9/00756 (Maestro 9).

Acknowledgments: We are grateful to Agnieszka Janiuk and Jina-Min Wang for very helpful recent discussions on the topic of slim disks.

Conflicts of Interest: The author declares no conflict of interest.

\section{References}

1. Abramowicz, M.A.; Czerny, B.; Lasota, J.P.; Szuszkiewicz, E. Slim accretion disks. Astron. J. 1988, 332, 646-658. [CrossRef]

2. Shakura, N.I.; Sunyaev, R.A. Black holes in binary systems. Observational appearance. Astron. Astrophys. 1973, 24, 337-355. 
3. Novikov, I.D.; Thorne, K.S. Astrophysics of black holes. In Black Holes (Les Astres Occlus); Dewitt, C., Dewitt, B.S., Eds.; Gordon and Breach Science Publishers: New York, NY, USA, 1973; pp. 343-450.

4. Abramowicz, M.A.; Fragile, P.C. Foundations of Black Hole Accretion Disk Theory. Living Rev. Relativ. 2013, 16. [CrossRef] [PubMed]

5. Frank, J.; King, A.; Raine, D.J. Accretion Power in Astrophysics, 3rd ed.; Cambridge University Press: Cambridge, UK, 2002; p. 398.

6. Abramowicz, M.A.; Chen, X.; Kato, S.; Lasota, J.P.; Regev, O. Thermal equilibria of accretion disks. Astrophys. J. Lett. 1995, 438, L37-L39. [CrossRef]

7. RóżaŃska, A.; Czerny, B. Vertical structure of the accreting two-temperature corona and the transition to an ADAF. Astron. Astrophys. 2000, 360, 1170-1186.

8. Liu, B.F.; Mineshige, S.; Meyer, F.; Meyer-Hofmeister, E.; Kawaguchi, T. Two-Temperature Coronal Flow above a Thin Disk. Astron. J. 2002, 575, 117-126. [CrossRef]

9. Hogg, J.D.; Reynolds, C.S. The Dynamics of Truncated Black Hole Accretion Disks. I. Viscous Hydrodynamic Case. Astron. J. 2017, 843, 80. [CrossRef]

10. Stoeger, W.R. Boundary-condition modification of the Novikov-Thorne accretion-disc models and velocity matching across the inner edge. Astron. Astrophys. 1976, 53, 267-274.

11. Paczynski, B.; Bisnovatyi-Kogan, G. A Model of a Thin Accretion Disk around a Black Hole. Acta Astron. 1981, 31, 283.

12. Loska, Z. Transonic disk accretion of barytropic gas onto black holes. Acta Astron. 1982, 32, $13-24$.

13. Muchotrzeb, B.; Paczynski, B. Transonic accretion flow in a thin disk around a black hole. Acta Astron. 1982, 32, 1-11.

14. Paczyński, B.; Wiita, P.J. Thick accretion disks and supercritical luminosities. Astron. Astrophys. 1980, $88,23-31$.

15. Muchotrzeb, B. Transonic accretion flow in a thin disk around a black hole. II. Acta Astron. 1983, 33, $79-87$.

16. Jaroszynski, M.; Abramowicz, M.A.; Paczynski, B. Supercritical accretion disks around black holes. Acta Astron. 1980, 30, 1-34.

17. Katz, J.I. X-rays from spherical accretion onto degenerate dwarfs. Astron. J. 1977, 215, 265-275. [CrossRef]

18. Begelman, M.C. Black holes in radiation-dominated gas-An analogue of the Bondi accretion problem. Mon. Not. R. Astron. Soc. 1978, 184, 53-67. [CrossRef]

19. Pringle, J.E.; Rees, M.J.; Pacholczyk, A.G. Accretion onto Massive Black Holes. Astron. Astrophys. 1973, 29, 179.

20. Lightman, A.P.; Eardley, D.M. Black Holes in Binary Systems: Instability of Disk Accretion. Astrophys. J. Lett. 1974, 187, L1. [CrossRef]

21. Shakura, N.I.; Sunyaev, R.A. A theory of the instability of disk accretion on to black holes and the variability of binary X-ray sources, galactic nuclei and quasars. Mon. Not. R. Astron. Soc. 1976, 175, 613-632. [CrossRef]

22. Lynden-Bell, D. Galactic Nuclei as Collapsed Old Quasars. Nature 1969, 223, 690-694. [CrossRef]

23. Sakimoto, P.J.; Coroniti, F.V. Accretion disk models for QSOs and active galactic nuclei - The role of magnetic viscosity. Astron. J. 1981, 247, 19-31. [CrossRef]

24. Sądowski, A.; Abramowicz, M.; Bursa, M.; Kluźniak, W.; Lasota, J.P.; Różańska, A. Relativistic slim disks with vertical structure. Astron. Astrophys. 2011,527, A17. [CrossRef]

25. RóżaŃska, A.; Czerny, B.; Życki, P.T.; Pojmański, G. Vertical structure of accretion discs with hot coronae in active galactic nuclei. Mon. Not. R. Astron. Soc. 1999, 305, 481-491. [CrossRef]

26. Wang, J.M.; Zhou, Y.Y. Self-similar Solution of Optically Thick Advection-dominated Flows. Astron. J. 1999, 516, 420-424. [CrossRef]

27. Szuszkiewicz, E.; Malkan, M.A.; Abramowicz, M.A. The Observational Appearance of Slim Accretion Disks. Astron. J. 1996, 458, 474. [CrossRef]

28. Straub, O.; Godet, O.; Webb, N.; Servillat, M.; Barret, D. Investigating the mass of the intermediate mass black hole candidate HLX-1 with the slimbh model. Astron. Astrophys. 2014, 569, A116. [CrossRef]

29. Castelló-Mor, N.; Kaspi, S.; Netzer, H.; Du, P.; Hu, C.; Ho, L.C.; Bai, J.M.; Bian, W.H.; Yuan, Y.F.; Wang, J.M. Unveiling slim accretion disc in AGN through X-ray and Infrared observations. Mon. Not. R. Astron. Soc. 2017, 467, 1209-1221. [CrossRef] 
30. Straub, O.; Bursa, M.; Sądowski, A.; Steiner, J.F.; Abramowicz, M.A.; Kluźniak, W.; McClintock, J.E.; Narayan, R.; Remillard, R.A. Testing slim-disk models on the thermal spectra of LMC X-3. Astron. Astrophys. 2011, 533, A67. [CrossRef]

31. Wang, J.M.; Qiu, J.; Du, P.; Ho, L.C. Self-shadowing Effects of Slim Accretion Disks in Active Galactic Nuclei: The Diverse Appearance of the Broad-line Region. Astron. J. 2014, 797, 65. [CrossRef]

32. Wang, J.M.; Du, P.; Hu, C.; Netzer, H.; Bai, J.M.; Lu, K.X.; Kaspi, S.; Qiu, J.; Li, Y.R.; Wang, F.; et al. Supermassive Black Holes with High Accretion Rates in Active Galactic Nuclei. II. The Most Luminous Standard Candles in the Universe. Astron. J. 2014, 793, 108. [CrossRef]

33. Marziani, P.; Bon, E.; Bon, N.; del Olmo, A.; Martínez-Aldama, M.; D'Onofrio, M.; Dultzin, D.; Negrete, C.; Stirpe, G. Quasars: From the Physics of Line Formation to Cosmology. Atoms 2019, 7, 18. [CrossRef]

34. Shen, Y.; Richards, G.T.; Strauss, M.A.; Hall, P.B.; Schneider, D.P.; Snedden, S.; Bizyaev, D.; Brewington, H.; Malanushenko, V.; Malanushenko, E.; et al. A Catalog of Quasar Properties from Sloan Digital Sky Survey Data Release 7. Astrophys. J. Suppl. 2011, 194, 45. [CrossRef]

35. Panda, S.; Czerny, B.; Adhikari, T.P.; Hryniewicz, K.; Wildy, C.; Kuraszkiewicz, J.; Śniegowska, M. Modeling of the Quasar Main Sequence in the Optical Plane. Astron. J. 2018, 866, 115. [CrossRef]

36. Collin, S.; Kawaguchi, T. Super-Eddington accretion rates in Narrow Line Seyfert 1 galaxies. Astron. Astrophys. 2004, 426, 797-808. [CrossRef]

37. Du, P.; Hu, C.; Lu, K.X.; Huang, Y.K.; Cheng, C.; Qiu, J.; Li, Y.R.; Zhang, Y.W.; Fan, X.L.; Bai, J.M.; et al. Supermassive Black Holes with High Accretion Rates in Active Galactic Nuclei. IV. H $\beta$ Time Lags and Implications for Super-Eddington Accretion. Astron. J. 2015, 806, 22. [CrossRef]

38. Negrete, C.A.; Dultzin, D.; Marziani, P.; Esparza, D.; Sulentic, J.W.; del Olmo, A.; Martínez-Aldama, M.L.; García López, A.; D’Onofrio, M.; Bon, N.; et al. Highly accreting quasars: The SDSS low-redshift catalog. Astron. Astrophys. 2018, 620, A118. [CrossRef]

39. Martínez-Aldama, M.L.; del Olmo, A.; Marziani, P.; Sulentic, J.W.; Negrete, C.A.; Dultzin, D.; D’Onofrio, M.; Perea, J. Extreme quasars at high redshift. Astron. Astrophys. 2018, 618, A179. [CrossRef]

40. Du, P.; Lu, K.X.; Zhang, Z.X.; Huang, Y.K.; Wang, K.; Hu, C.; Qiu, J.; Li, Y.R.; Fan, X.L.; Fang, X.E.; et al. Supermassive Black Holes with High Accretion Rates in Active Galactic Nuclei. V. A New Size-Luminosity Scaling Relation for the Broad-line Region. Astron. J. 2016, 825, 126. [CrossRef]

41. Du, P.; Zhang, Z.X.; Wang, K.; Huang, Y.K.; Zhang, Y.; Lu, K.X.; Hu, C.; Li, Y.R.; Bai, J.M.; Bian, W.H.; et al. Supermassive Black Holes with High Accretion Rates in Active Galactic Nuclei. IX. 10 New Observations of Reverberation Mapping and Shortened H $\beta$ Lags. Astron. J. 2018, 856, 6. [CrossRef]

42. Janiuk, A.; Czerny, B. On different types of instabilities in black hole accretion discs: implications for X-ray binaries and active galactic nuclei. Mon. Not. R. Astron. Soc. 2011, 414, 2186-2194. [CrossRef]

43. Meyer, F.; Meyer-Hofmeister, E. On the Elusive Cause of Cataclysmic Variable Outbursts. Astron. Astrophys. 1981, 104, L10.

44. Smak, J. Outbursts of dwarf novae. Publ. Astron. Soc. Pac. 1984, 96, 5-18. [CrossRef]

45. Nayakshin, S.; Rappaport, S.; Melia, F. Time-dependent Disk Models for the Microquasar GRS $1915+105$. Astron. J. 2000, 535, 798-814. [CrossRef]

46. Janiuk, A.; Czerny, B.; Siemiginowska, A. Radiation Pressure Instability as a Variability Mechanism in the Microquasar GRS 1915+105. Astrophys. J. Lett. 2000, 542, L33-L36. [CrossRef]

47. Janiuk, A.; Czerny, B.; Siemiginowska, A. Radiation Pressure Instability Driven Variability in the Accreting Black Holes. Astron. J. 2002, 576, 908-922. [CrossRef]

48. Janiuk, A.; Czerny, B. Accreting corona model of the X-ray variability in soft state X-ray binaries and active galactic nuclei. Astron. Astrophys. 2007, 466, 793-803. [CrossRef]

49. Czerny, B.; Siemiginowska, A.; Janiuk, A.; Nikiel-Wroczyński, B.; Stawarz, Ł. Accretion Disk Model of Short-Timescale Intermittent Activity in Young Radio Sources. Astron. J. 2009, 698, 840-851. [CrossRef]

50. Grzędzielski, M.; Janiuk, A.; Czerny, B.; Wu, Q. Modified viscosity in accretion disks. Application to Galactic black hole binaries, intermediate mass black holes, and active galactic nuclei. Astron. Astrophys. 2017, 603, A110. [CrossRef]

51. Grzędzielski, M.; Janiuk, A.; Czerny, B. Local Stability and Global Instability in Iron-opaque Disks. Astron. J. 2017, 845, 20. [CrossRef]

52. Gu, W.M.; Lu, J.F. A Note on the Slim Accretion Disk Model. Astron. J. 2007, 660, 541-545. [CrossRef] 
53. Lasota, J.P.; Vieira, R.S.S.; Sadowski, A.; Narayan, R.; Abramowicz, M.A. The slimming effect of advection on black-hole accretion flows. Astron. Astrophys. 2016, 587, A13. [CrossRef]

54. Janiuk, A.; Czerny, B. Time-delays between the soft and hard X-ray bands in GRS 1915+105. Mon. Not. R. Astron. Soc. 2005, 356, 205-216. [CrossRef]

55. Baldi, R.D.; Capetti, A.; Giovannini, G. Pilot study of the radio-emitting AGN population: The emerging new class of FR 0 radio-galaxies. Astron. Astrophys. 2015, 576, A38. [CrossRef]

56. Wu, Q.; Czerny, B.; Grzedzielski, M.; Janiuk, A.; Gu, W.M.; Dong, A.J.; Cao, X.F.; You, B.; Yan, Z.; Sun, M.Y. The Universal Heartbeat Oscillations in Black Hole Systems Across the Mass-scale. Astron. J. 2016, 833, 79. [CrossRef]

57. Szuszkiewicz, E. Slim accretion discs with different viscosity prescriptions. Mon. Not. R. Astron. Soc. 1990, 244, 377-383.

58. Janiuk, A.; Grzedzielski, M.; Capitanio, F.; Bianchi, S. Interplay between heartbeat oscillations and wind outflow in microquasar IGR J17091-3624. Astron. Astrophys. 2015, 574, A92. [CrossRef]

59. Ohsuga, K.; Mori, M.; Nakamoto, T.; Mineshige, S. Supercritical Accretion Flows around Black Holes: Two-dimensional, Radiation Pressure-dominated Disks with Photon Trapping. Astron. J. 2005, 628, 368-381. [CrossRef]

60. Balbus, S.A.; Hawley, J.F. A powerful local shear instability in weakly magnetized disks. I—Linear analysis. II-Nonlinear evolution. Astron. J. 1991, 376, 214-233. [CrossRef]

61. Turner, N.J. On the Vertical Structure of Radiation-dominated Accretion Disks. Astrophys. J. Lett. 2004, 605, L45-L48. [CrossRef]

62. Hirose, S.; Krolik, J.H.; Blaes, O. Radiation-Dominated Disks are Thermally Stable. Astron. J. 2009, 691, 16-31. [CrossRef]

63. Jiang, Y.F.; Stone, J.M.; Davis, S.W. On the Thermal Stability of Radiation-dominated Accretion Disks. Astron. J. 2013, 778, 65. [CrossRef]

64. Jiang, Y.F.; Davis, S.W.; Stone, J.M. Iron Opacity Bump Changes the Stability and Structure of Accretion Disks in Active Galactic Nuclei. Astron. J. 2016, 827, 10. [CrossRef]

65. Svensson, R.; Zdziarski, A.A. Black hole accretion disks with coronae. Astron. J. 1994, 436, 599-606. [CrossRef]

66. Zycki, P.T.; Collin-Souffrin, S.; Czerny, B. Accretion Discs with Accreting Coronae in Active Galactic Nuclei-Part One-Solutions in Hydrostatic Equilibrium. Mon. Not. R. Astron. Soc. 1995, 277, 70. [CrossRef]

67. Chakrabarti, S.; Titarchuk, L.G. Spectral Properties of Accretion Disks around Galactic and Extragalactic Black Holes. Astron. J. 1995, 455, 623. [CrossRef]

68. Czerny, B.; Nikołajuk, M.; Różańska, A.; Dumont, A.M.; Loska, Z.; Zycki, P.T. Universal spectral shape of high accretion rate AGN. Astron. Astrophys. 2003, 412, 317-329. [CrossRef]

69. Rajesh, S.R.; Mukhopadhyay, B. Two-temperature accretion around rotating black holes: a description of the general advective flow paradigm in the presence of various cooling processes to explain low to high luminous sources. Mon. Not. R. Astron. Soc. 2010, 402, 961-984. [CrossRef]

70. Begelman, M.C.; Armitage, P.J.; Reynolds, C.S. Accretion Disk Dynamo as the Trigger for X-Ray Binary State Transitions. Astron. J. 2015, 809, 118. [CrossRef]

71. Begelman, M.C.; Silk, J. Magnetically elevated accretion discs in active galactic nuclei: broad emission-line regions and associated star formation. Mon. Not. R. Astron. Soc. 2017, 464, 2311-2317. [CrossRef]

72. Dexter, J.; Begelman, M.C. Extreme AGN variability: Evidence of magnetically elevated accretion? Mon. Not. R. Astron. Soc. 2019, 483, L17-L21. [CrossRef]

73. Gronkiewicz, D.; Różańska, A. Warm and thick corona for magnetically supported disk in GBHB. arXiv 2019, arXiv:1903.03641.

74. Fragile, P.C.; Sądowski, A. On the decay of strong magnetization in global disc simulations with toroidal fields. Mon. Not. R. Astron. Soc. 2017, 467, 1838-1843. [CrossRef]

75. Janiuk, A.; Misra, R. Stabilization of radiation pressure dominated accretion disks through viscous fluctuations. Astron. Astrophys. 2012, 540, A114. [CrossRef]

76. Ahmad, N.; Misra, R.; Iqbal, N.; Maqbool, B.; Hamid, M. Modeling the response of a standard accretion disc to stochastic viscous fluctuations. New Astron. 2018, 58, 84-89. [CrossRef]

77. Narayan, R.; Igumenshchev, I.V.; Abramowicz, M.A. Magnetically Arrested Disk: An Energetically Efficient Accretion Flow. Publ. Astron. Soc. Jpn. 2003, 55, L69-L72. [CrossRef] 
78. Mondal, T.; Mukhopadhyay, B. Ultraluminous X-ray sources as magnetically powered sub-Eddington advective accretion flows around stellar mass black holes. Mon. Not. R. Astron. Soc. 2019, 482, L24-L28. [CrossRef]

79. Jiang, Y.F.; Blaes, O.; Stone, J.; Davis, S.W. Global Radiation Magneto-hydrodynamic Simulations of Sub-Eddington Accretion Disks around Supermassive Black Holes. arXiv 2019, arXiv:1904.01674.

80. Ghoreyshi, S.M.; Shadmehri, M. The local stability of the magnetized advection-dominated discs with the radial viscous force. Mon. Not. R. Astron. Soc. 2018, 476, 4830-4839. [CrossRef]

81. McKinney, J.C.; Chluba, J.; Wielgus, M.; Narayan, R.; Sadowski, A. Double Compton and Cyclo-Synchrotron in Super-Eddington Discs, Magnetized Coronae, and Jets. Mon. Not. R. Astron. Soc. 2017, 467, 2241-2265. [CrossRef]

82. Sadowski, A.; Abramowicz, M.A.; Bursa, M.; Kluźniak, W.; Różańska, A.; Straub, O. Vertical dissipation profiles and the photosphere location in thin and slim accretion disks. Astron. Astrophys. 2009, 502, 7-13. [CrossRef]

83. Liu, B.F.; Taam, R.E.; Qiao, E.; Yuan, W. Centrally Concentrated X-Ray Radiation from an Extended Accreting Corona in Active Galactic Nuclei. Astron. J. 2017, 847, 96. [CrossRef]

84. De Gouveia Dal Pino, E.M.; Piovezan, P.P.; Kadowaki, L.H.S. The role of magnetic reconnection on jet/accretion disk systems. Astron. Astrophys. 2010, 518, A5. [CrossRef]

85. Hawley, J.F.; Balbus, S.A. Anomalous Viscosity in Accretion Disks. In Wild Stars in the Old West; Howell, S., Kuulkers, E., Woodward, C., Eds.; Astronomical Society of the Pacific Conference Series; University of Virginia: Charlottesville, VR, USA, 1998; Volume 137, p. 273.

86. Kubota, A.; Done, C. Modeling the spectral energy distributions of super-Eddington quasars. arXiv 2019, arXiv:1905.02920.

(C) 2019 by the author. Licensee MDPI, Basel, Switzerland. This article is an open access article distributed under the terms and conditions of the Creative Commons Attribution (CC BY) license (http:/ / creativecommons.org/licenses/by/4.0/). 